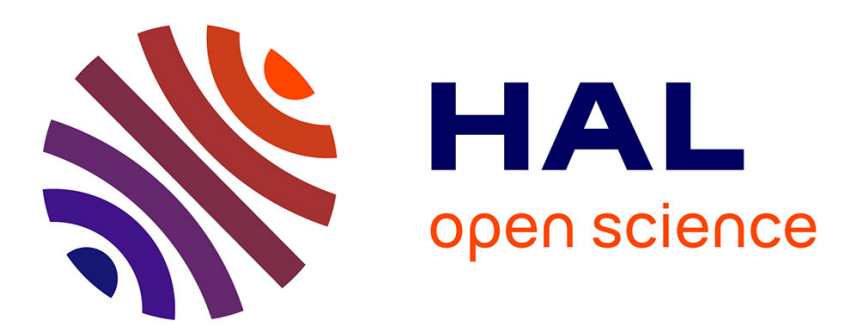

\title{
A Fast Wavelet-to-Wavelet Propagation Method for the Simulation of Long-Range Propagation in Low Troposphere
}

\author{
Hang Zhou, Alexandre Chabory, Rémi Douvenot
}

\section{- To cite this version:}

Hang Zhou, Alexandre Chabory, Rémi Douvenot. A Fast Wavelet-to-Wavelet Propagation Method for the Simulation of Long-Range Propagation in Low Troposphere. IEEE Transactions on Antennas and Propagation, 2022, 70 (3), pp.2137-2148. 10.1109/TAP.2021.3118821 . hal-03381905

\section{HAL Id: hal-03381905 https://hal.science/hal-03381905}

Submitted on 18 Oct 2021

HAL is a multi-disciplinary open access archive for the deposit and dissemination of scientific research documents, whether they are published or not. The documents may come from teaching and research institutions in France or abroad, or from public or private research centers.
L'archive ouverte pluridisciplinaire HAL, est destinée au dépôt et à la diffusion de documents scientifiques de niveau recherche, publiés ou non, émanant des établissements d'enseignement et de recherche français ou étrangers, des laboratoires publics ou privés. 


\title{
A Fast Wavelet-to-Wavelet Propagation Method for the Simulation of Long-Range Propagation in Low Troposphere
}

\author{
Hang Zhou, Alexandre Chabory, and Rémi Douvenot
}

\begin{abstract}
As an alternative method to the discrete split-step Fourier method, the split-step wavelet method has recently been derived. This method based on a wavelet decomposition of the field is efficient and accurate in simulating the long-range propagation of electromagnetic waves in the low troposphere. In this paper, to further improve the computational efficiency and take full advantage of wavelet characteristics, a waveletto-wavelet propagation method is proposed. The propagation, variable refractivity, and apodization are accounted in the wavelet domain. The computational complexity is reduced, since no transforms are performed between wave fields and wavelet coefficients. This method works well for the propagation over a planar ground with a constant impedance. When a variable impedance ground condition and/or an irregular relief are considered, a hybridization with the split-step wavelet method is proposed. These methods are tested and validated by means of numerical experiments, showing very good efficiency and accuracy.
\end{abstract}

Index Terms-Wavelet, propagation, electromagnetic wave, split-step method, refractivity, impedance ground, relief

\section{INTRODUCTION}

$\mathbf{T}$ HE prediction of the long-range propagation of electromagnetic waves is a main issue for radio frequencies. The parabolic equation method (PE) [1] is one of the most widely used methods. It can be solved iteratively in increasing distances by the finite-differences (FD) [2] and the split-step Fourier methods (SSF) [3] [4]. An inhomogeneous atmosphere, a ground condition, and an irregular relief can be accounted in these methods [5]. Between both methods, the SSF is numerically more efficient for large-scale problems, since it permits larger grid increments. At each step in SSF, the wave is transformed from the spatial domain to the spectral domain by means of a Fourier transform. Lately, Zhou et al. [6] [7] have proposed the discrete SSF method (DSSF). This improvement of SSF brings self-consistency [8] [9], with which numerical spurious solutions due to the a-posteriori discretization are avoided.

Other methods have also been proposed for propagation modeling, in which the electromagnetic wave is decomposed into elementary functions and the propagation is realized by the analytic formulation of beam propagation. For the Gaussian beams methods, the wave field is decomposed into

Sino-European Institute of Aviation Engineering, Civil Aviation University of China, Tianjin, China,

Ecole Nationale de l'Aviation Civile, TELECOM-EMA, Toulouse, France,

Toulouse University, Toulouse, France,

e-mail: h-zhoudcauc.edu.cn,

e-mail: chabory@recherche.enac.fr,

e-mail: remi.douvenoteenac.fr. the Gaussian functions [10] [11]. The Gabor-based beam algorithms and frame-based beam summation methods are also utilized in various applications involving radiation and scattering in complex environments [12]-[15]. In these methods, refraction effects can be modeled, whereas the relief at grazing incidence with large variations can not be accounted [2] [16] [17].

Here, we use the wavelet decomposition for the propagation modeling. The multi-resolution wavelets are wavelike oscillation functions constructed by dilations and/or translations from a mother wavelet and a scaling function [18]. A wave can be decomposed on these wavelets by means of the fast wavelet transform. The wavelet-based methods have been firstly used in the method of moments to improve the efficiency [19]. Then, a wavelet-based algorithm to solve PE has been proposed in [20] [21]. This method can model the propagation as accurately as DSSF. However, its computational complexity is the same as DSSF, since a Fourier transform is required in the computation.

In order to improve the efficiency of the long-range propagation method, a split-step wavelet method (SSW) has been presented in [22]. It is an alternative solution to DSSF. At each range step, direct and inverse wavelet transforms are performed. The propagation is computed in the wavelet domain by means of a multiplication by a pre-computed propagation matrix. The SSW is more efficient than DSSF while keeping a good accuracy. This is due to the high efficiency of wavelets for data compression and the low complexity of the fast wavelet transforms (FWT). However, the refractivity, relief, and apodization are accounted in the spatial domain. This means that the transforms between wave fields and wavelet coefficients are required at each marching step, similarly to DSSF.

In this work, to further improve the efficiency of SSW, a new wavelet-based method is proposed. The main idea is to perform all the propagation in the wavelet domain. This method is denoted as the wavelet-to-wavelet propagation method (WWP). Since no transform is performed during the propagation, the computation time of WWP is shorter than SSW. Furthermore, the refractivity and apodization is accounted in the wavelet domain and the relief is considered by a hybridization method with SSW.

In Section II, a brief introduction of the wavelet decomposition and the split-step wavelet method is presented. In Section III, the proposed wavelet-to-wavelet propagation method is introduced for the wave propagation over a planar ground 
with a constant surface impedance. In Section IV, the method for a variable impedance ground and/or an irregular relief is introduced. In Section V, the different methods and their computational complexities are summarized. Finally, tests and validations are performed in Section VI.

\section{BRIEF INTRODUCTION OF WAVELET DECOMPOSITION AND SPLIT-STEP WAVELET METHOD}

\section{A. Configuration and discretization}

We assume an $e^{j \omega t}$ time-dependence of the fields, with $\omega$ the angular frequency. The aim is to simulate the propagation in a vertical plane $(x, z)$ with $y$ the direction of invariance. The source is assumed to be located at $x<0$ and the field $u(0, z)$ is known. The propagation is computed in the region $x>0, z \geq 0$. An impedance ground condition is assumed. The fields can be decomposed in a transverse electric (TE) and a transverse magnetic (TM) components with respect to the vertical direction $z$.

For numerical reasons, the computation domain is of finite size and discretized. The computation domain is limited to $x \in\left[0, x_{\max }\right]$ and $z \in\left[0, z_{\max }\right]$. At the ground $z=0$, an impedance ground condition is accounted. At $z=z_{\max }$, the top condition is assumed the same as the ground condition. An apodization is then applied to remove the reflections over the top boundary.

The discretization steps are $\Delta x$ and $\Delta z$. The computation domain is discretized by a uniform grid, such as

$$
\begin{array}{lll}
x=p_{x} \Delta x & \text { for } & p_{x}=\left\{0, \ldots, N_{x}\right\}, \\
z=p_{z} \Delta z & \text { for } \quad p_{z}=\left\{0, \ldots, N_{z}\right\},
\end{array}
$$

with $N_{x}=x_{\max } / \Delta x$ and $N_{z}=z_{\max } / \Delta z$.

The field of the position $\left(p_{x} \Delta x, p_{z} \Delta z\right)$ is represented by $u_{p_{x}, p_{z}}$ and the vector $u\left(p_{x} \Delta x, \cdot\right)$ is denoted as $u_{p_{x}}$ in the following parts.

\section{B. Multiscale wavelet decomposition}

A wavelet is a wavelike oscillation function localized in both space and frequency domains. A discrete function can be represented as a linear combination of multiscale elementary functions by applying the discrete wavelet transform (DWT) [23]. These elementary functions can be regarded by being constructed by dilations and translations of a scaling function $\phi$ and a mother wavelet $\psi$ [23], as illustrated in Fig. 1a and 1b. Assuming the maximum decomposition level $L \in \mathbb{N}^{+}$, the scaling functions $\left(\phi_{L, p}\right)_{p \in\left[0, N_{p}(L)-1\right]}$ and the wavelet functions $\left(\psi_{l, p}\right)_{l \in[1, L], p \in\left[0, N_{p}(l)-1\right]}$, with $N_{p}(l)=$ $N_{z} / 2^{l}$, form an orthonormal basis to the space of the discrete functions with a length of $N_{z}$, as illustrated in Fig. 1c. Therefore, a discrete function $u[\cdot]$ can be represented by

$$
u[\cdot]=\sum_{l=0}^{L} \sum_{p=0}^{N_{p}(l)-1} U_{(l, p)} \chi_{l, p}[\cdot],
$$

where the scaling function and the multiscale wavelet functions are expressed as

$$
\chi_{l, p}[\cdot]= \begin{cases}\phi_{L, p}[\cdot] & \text { for } \quad l=0 \\ \psi_{l, p}[\cdot] & \text { for } \quad l \in[1, L]\end{cases}
$$

The wavelet coefficients are represented by the vector $U$. The total size of $U$ is $N_{z}$. Each element of $U$, represented by double indices $(l, p)$, are defined by

$$
U_{(l, p)}= \begin{cases}a_{L}[p] & \text { for } \quad l=0, p \in\left[0, N_{p}(L)-1\right], \\ d_{l}[p] & \text { for } \quad l \in[1, L], p \in\left[0, N_{p}(l)-1\right],\end{cases}
$$

with $a_{L}[p]$ the approximation coefficients and $d_{l}[p]$ the detail coefficients [23]. For computing these coefficients, a fast algorithm for the discrete wavelet transform, the fast wavelet transform (FWT), can be applied. Its computational complexity is of order of $O\left(N_{z}\right)$, which is better than the one of fast Fourier transform (FFT), i.e., $O\left(N_{z} \log N_{z}\right)$ [24].

The wavelet transform is a very efficient tool for data compression. By forcing to 0 the coefficients that are close to zero, a signal can be approximated by a sparse set of coefficients within a chosen accuracy. The compressed vector $\tilde{U}$ is

$$
\tilde{U}_{(l, p)}=\left\{\begin{array}{l}
0 \text { for }\left|U_{(l, p)}\right| \leq V_{\mathrm{s}}, \\
U_{(l, p)} \text { for }\left|U_{(l, p)}\right|>V_{\mathrm{s}},
\end{array}\right.
$$

with $V_{\mathrm{s}}$ the compression threshold. Generally, $\tilde{U}$ is a sparse vector and the number of non-zero coefficients $N_{\mathrm{s}}$ is typically much smaller than the original signal size $N_{z}$.

\section{Split-step wavelet method}

For the SSW method, which is introduced in [22], the propagation of the field $u$ from $p_{x} \Delta x$ to $\left(p_{x}+1\right) \Delta x$ is computed by

$$
u_{p_{x}+1}=\mathbf{L H R W}^{-1} \mathbf{M}^{\mathrm{f}} \mathbf{C W} u_{p_{x}},
$$

where $\mathbf{W}$ and $\mathbf{W}^{-1}$ are the direct and inverse fast wavelet transforms. The operator $\mathbf{C}$ corresponds to data compression with the threshold value $V_{s} . \mathbf{M}^{\mathrm{f}}$ is the free-space propagation matrix, containing all the pre-computed wavelet-to-wavelet propagations. Thus, $\mathbf{M}^{\mathrm{f}}$ represents the propagation operator of the wave in the wavelet domain. The propagation on $\Delta x$ is computed by multiplying the compressed wavelet coefficients by $\mathbf{M}^{\mathrm{f}}$. Besides, $\mathbf{R}$ corresponds to the phase screen which accounts for the refractivity. Then, an apodization $\mathbf{H}$ is applied. The relief is considered by the operator $\mathbf{L}$. These steps are repeated for the wave propagation at increasing distances.

In Section 4.2 of [22], we have introduced an efficient filling method for $\mathbf{M}^{\mathrm{f}}$. The fast filling algorithm can be achieved, because the wavelets have the property to be shift-invariant on the same decomposition level. In total, only $L+1$ DSSF propagations and $2^{L}$ FWT are necessary to calculate the entire matrix $\mathbf{M}^{\mathrm{f}}$, with $L$ the maximum decomposition level.

\section{WAVELET-TO-WAVELET PROPAGATION METHOD}

For simplicity of presentation, firstly, the fundamental wavelet-to-wavelet propagation method without considering the ground is presented, denoted as WWP. Then, a phase screen method applied on the wavelet coefficients is introduced. Finally, a WWP-M method is developed to simulate the reflections over a constant impedance planar ground. 


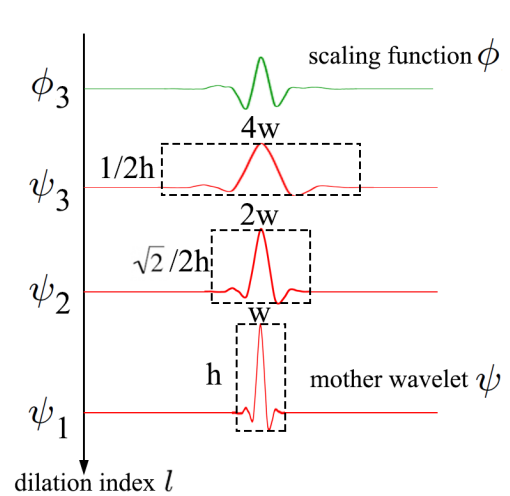

(a) Wavelets of different levels are derived by dilating the scaling function $\phi$ and the mother wavelet $\psi$.

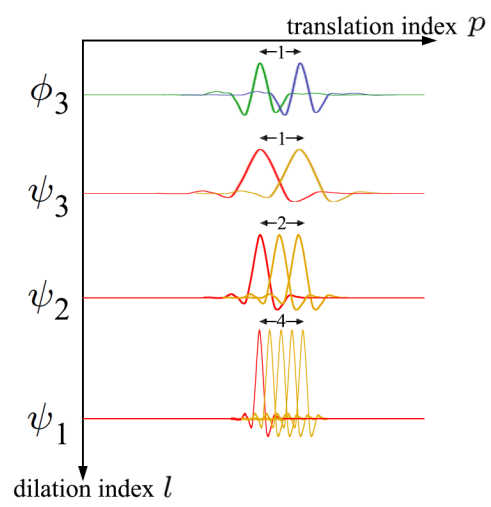

(b) Wavelets of the same level are derived by translations.

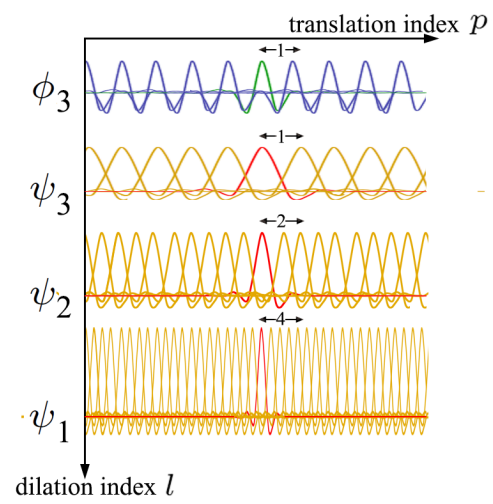

(c) All the multiscale wavelets form an orthonormal wavelet basis.

Fig. 1. Illustrations of the multiscale wavelets with the maximum decomposition level $L=3$.

\section{A. Overview of $W W P$}

The computation steps for the WWP method are illustrated in Fig. 2. As the initialization, the field at $x=0$, i.e., $u_{0}$, is transformed to its wavelet coefficients $U_{0}$ by applying FWT.

The propagation of the wavelet coefficients $U$ is computed marching on in distances. The propagation on one step in distance $\Delta x$ of the wavelet coefficients at $p_{x} \Delta x$ with $p_{x} \in$ $\left[0, N_{x}-1\right]$, denoted as $U_{p_{x}}$, is simulated step-by-step as follows:

1) A data compression operator $\mathbf{C}$ is applied on $U_{p_{x}}$ with a chosen threshold value $V_{\mathrm{s}}$. This operator is introduced in [22]. The compressed coefficients $\tilde{U}$ at $p_{x} \Delta x$ are given by

$$
\tilde{U}_{p_{x}}=\mathbf{C} U_{p_{x}} .
$$

2) The propagation is computed by means of multiplying by a pre-computed propagation matrix $\tilde{\mathbf{M}}$. The wavelet coefficients $U^{\mathrm{p}}$ at $\left(p_{x}+1\right) \Delta x$ are

$$
U_{p_{x}+1}^{\mathrm{p}}=\tilde{\mathbf{M}} \tilde{U}_{p_{x}} .
$$

The apodization is accounted in $\tilde{\mathbf{M}}$, as detailed in Section III-B. This matrix is sparse after being compressed by a threshold $V_{\mathrm{P}}$. The elements of $\tilde{\mathbf{M}}$, i.e., $\tilde{\mathbf{M}}_{(l, p),\left(l^{\prime}, p^{\prime}\right)}$, satisfy

$$
U_{p_{x}+1,(l, p)}^{\mathrm{p}}=\sum_{l^{\prime}, p^{\prime}} \tilde{\mathbf{M}}_{(l, p),\left(l^{\prime}, p^{\prime}\right)} \tilde{U}_{p_{x},\left(l^{\prime}, p^{\prime}\right)} .
$$

3) A phase screen directly applied on the wavelet coefficients, denoted as $\mathbf{R}^{\mathrm{c}}$, is used to consider the variable refractivity. This operator will be introduced in Section III-C.

$$
U_{p_{x}+1}=\mathbf{R}^{\mathrm{c}} U_{p_{x}+1}^{\mathrm{p}}
$$

As a conclusion, the propagation of $U_{p_{x}}$ on $\Delta x$ is

$$
U_{p_{x}+1}=\mathbf{R}^{\mathrm{c}} \tilde{\mathbf{M}} \mathbf{C} U_{p_{x}} .
$$

These steps are iteratively repeated to simulate the propagation. The field at the final step is obtained by applying the inverse FWT on the coefficients.

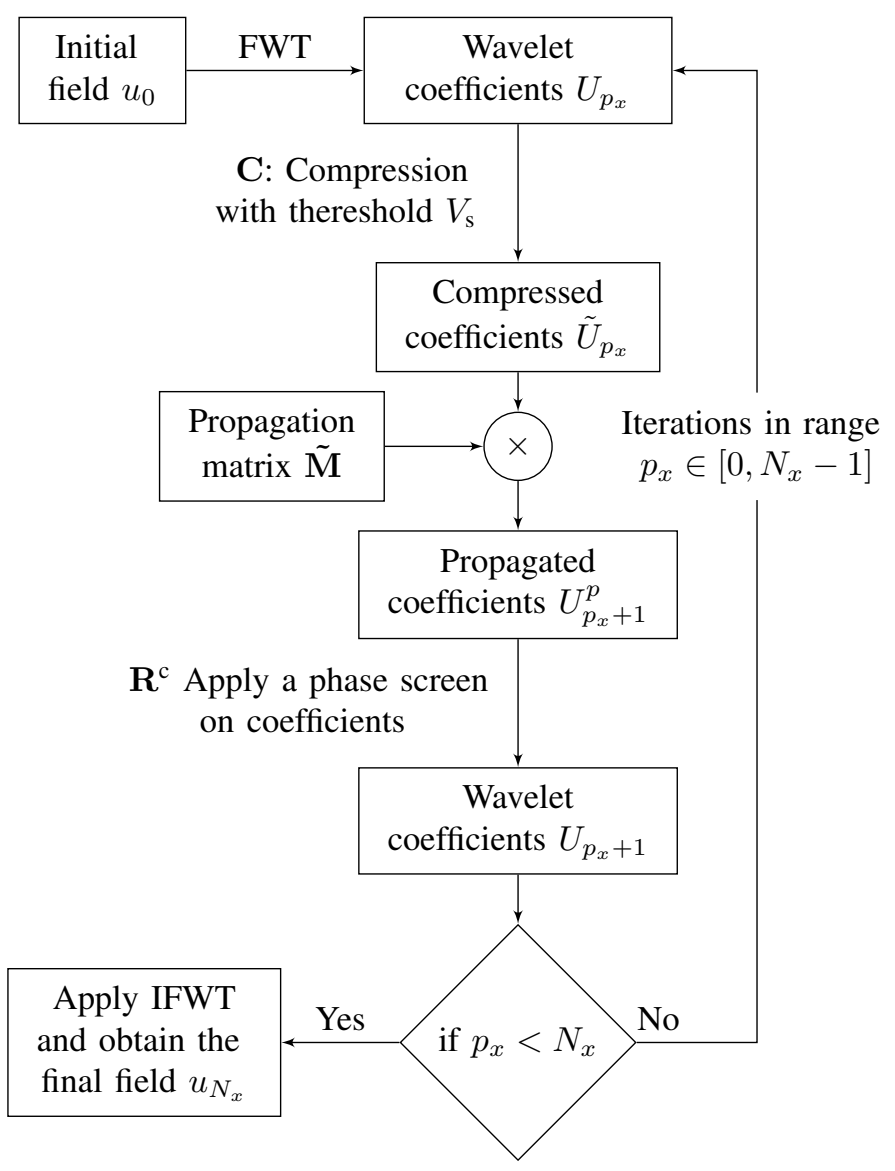

Fig. 2. Overview of WWP for the wave propagation in an inhomogeneous atmosphere.

\section{B. Propagation matrix $\tilde{\mathbf{M}}$}

The propagation matrix $\tilde{\mathbf{M}}$, mentioned in Section III-A, accounts for the wave propagation on one step $\Delta x$ with the apodization. First, the propagation matrix for free-space propagation is introduced for the sake of a simple presentation, which is denoted as $\mathbf{M}^{\mathrm{f}}$. Then, the apodization is included, so that $\tilde{\mathbf{M}}$ is derived. 


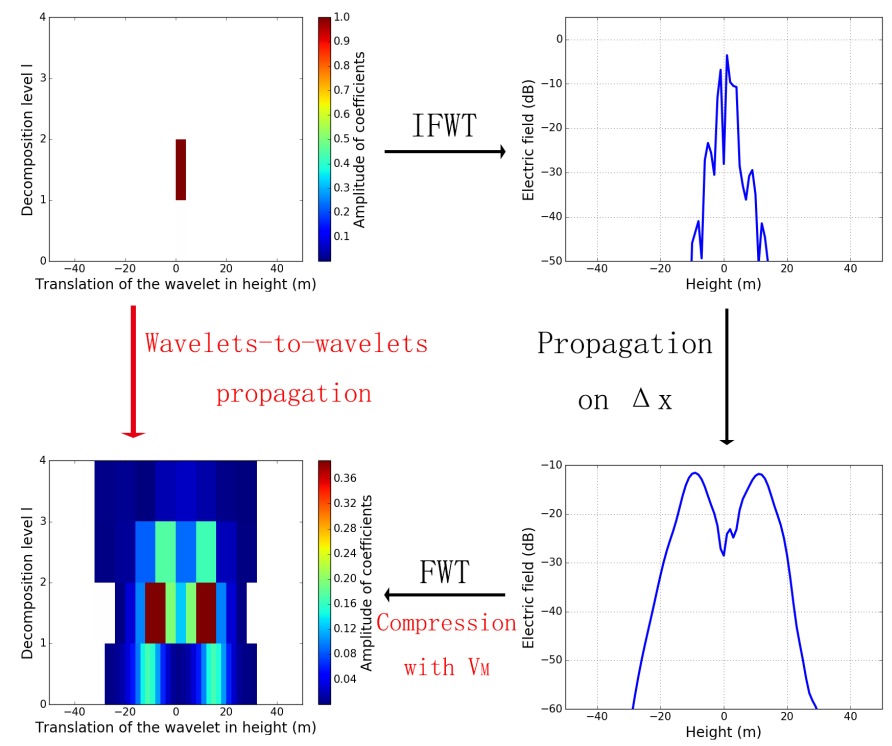

Fig. 3. Illustration of the free-space propagation of one wavelet, giving the relation of one coefficient to the propagated coefficients.

1) Free-space propagation matrix $\mathbf{M}^{f}$ : No ground is considered and the matrix $\mathbf{M}^{\mathrm{f}}$ records the information of freespace propagations of wavelets. The elements of the matrix are $M_{(l, p)\left(l^{\prime}, p^{\prime}\right)}^{\mathrm{f}}$, where $l^{\prime}$ and $l$ correspond to the wavelet levels of the coefficients before and after propagation, respectively. The indices $p^{\prime}$ and $p$ correspond to the position indices along the vertical axis before and after propagation, respectively.

The construction of $\mathbf{M}^{\mathrm{f}}$ is illustrated in Fig. 3 and introduced as follows:

- Firstly, only one wavelet coefficient $U_{\left(l^{\prime}, p^{\prime}\right)}$ is considered and set to 1 . All other coefficients are set to zero. The coefficient $U_{\left(l^{\prime}, p^{\prime}\right)}$ is transformed to field representation $\chi_{l^{\prime}, p^{\prime}}(0, \cdot)$, by applying an IFWT.

- The wavelet $\chi_{l^{\prime}, p^{\prime}}(0, \cdot)$ is propagated on $\Delta x$. The propagated wavelets $\chi_{l^{\prime}, p^{\prime}}(\Delta x)$ are obtained by means of DSSF [7] (Any other propagation methods be used as well).

- The propagated wavelet coefficients are obtained by applying a FWT on $\chi_{l^{\prime}, p^{\prime}}(\Delta x, \cdot)$ and a compression.

- One column of the propagation matrix, $\mathbf{M}_{(\cdot, \cdot)\left(l^{\prime}, p^{\prime}\right)}^{\mathrm{f}}$, contains the propagated coefficients of $\chi_{l^{\prime}, p^{\prime}}(\Delta x, \cdot)$.

The elements $M_{(l, p)\left(l^{\prime}, p^{\prime}\right)}$ for all $l, l^{\prime}, p, p^{\prime}$ are obtained by a fast filling method, exploiting the translation properties of $M^{\mathrm{f}}$, as introduced in [22]. Only the propagation of one wavelet for each level needs to be computed. The other propagated coefficients could be derived by translations. Thus, $L+1$ wavelet propagations are required to be simulated in total. The computation burden is quite low for filling in the matrix. Note that this matrix could be replaced by a library of local propagators, which slightly modify the propagation [25] [26].

2) Apodization included in the propagation matrix $\tilde{\mathbf{M}}$ : The wavelets are localized in space. For one wavelet with the dilation and translation indices $(l, p)$, its vertical position is

$$
z(l, p)= \begin{cases}2^{l} p & \text { for } \quad l \in\{1, \ldots, L\} \\ 2^{L} p & \text { for } \quad l=0 .\end{cases}
$$

For the wave propagation without ground, to remove the reflections over the top and bottom boundaries, two absorbing layers are assumed in the domains $z \in\left[-z_{\max }, 0\right]$ and $z \in\left[z_{\max }, 2 z_{\max }\right]$. For example, for the absorbing layer of the top boundary, the apodization amounts to a term-by-term multiplication by a diagonal operator $\mathbf{H}^{\mathrm{c}}$. Its elements are defined by

$\mathbf{H}_{(l, p)}^{\mathrm{c}}= \begin{cases}1 & \text { for } z(l, p) \leq z_{\max }, \\ \frac{1+\cos \left(\pi\left(\frac{z(l, p)-z_{\max }}{z_{\max }}\right)\right)}{2} & \text { for } z_{\max }<z(l, p) \leq 2 z_{\max } .\end{cases}$

This equation amounts to directly applying the apodization layer on the wavelet coefficients.

The propagation matrix including the apodization is computed by $\tilde{\mathbf{M}}_{(\cdot, \cdot),\left(l^{\prime}, p^{\prime}\right)}=\mathbf{H}^{\mathrm{c}} \mathbf{M}_{(\cdot, \cdot),\left(l^{\prime}, p^{\prime}\right)}^{\mathrm{f}}$. Since the absorbing window is applied directly on the coefficients, one may think that it is different to the canonical absorbing window applied on the fields. Indeed, since the attenuation coefficients in (13) are multiplied term-by-term on the wavelet coefficients, this means that one attenuation remains constant on the support of the corresponding wavelet. This leads to a difference compared to the absorbing window on fields. To mitigate this problem, the parameters of the wavelets are carefully chosen, such that the support of the most dilated wavelet remains small w.r.t. the size of the apodization layer. Tests with different wavelet parameters are presented in Section VI-A.

The propagation matrix $\tilde{\mathbf{M}}$ is precomputed before the computation for wave propagation. This matrix is recorded as preacquired data, used for all the simulations with the same parameters.

\section{Phase screen}

The phase screen operator $\mathbf{R}^{\mathrm{c}}$, mentioned in Section III-A, is introduced in this section. The phase screen method is used to consider a variable refractivity in DSSF and SSW. In WWP, this method is applied on the wavelet coefficients, which is possible due to the localization property of the wavelet.

For each coefficient $U_{(l, p)}$, its vertical position $z(l, p)$ is given in (12). Here, an approximation is assumed that the refractivity is constant over the width of each wavelet. The error remains small when the most dilated wavelets are of narrow width, which is the same as the discussion in Section III-B2. The error of this assumption depends on the wavelet parameters and gradient of refractivity, which is tested in Section VI-B2.

The operator $\mathbf{R}^{\mathrm{c}}$ amounts to a term-by-term multiplication by $U_{p_{x}}(l, p)$ by a phase screen $R_{p_{x}}^{\mathrm{c}}(l, p)$, defined by

$$
R_{p_{x}}^{\mathrm{c}}(l, p)=\exp \left(\frac{n\left(p_{x} \Delta x, z(l, p)\right)-1}{2}\right) .
$$

This corresponds to the phase screen used in the wide-angle DSSF algorithm [27]. 


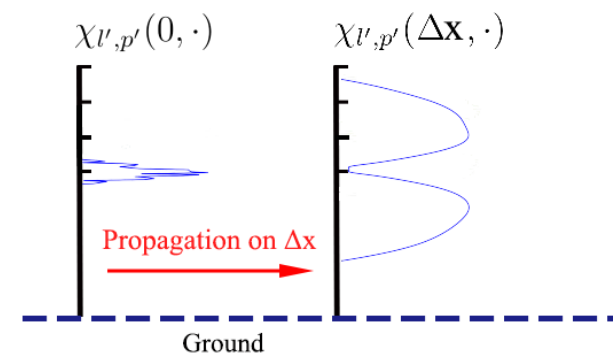

(a) Wavelet propagated in free space.

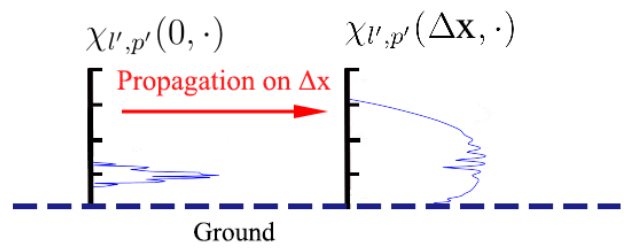

(b) Wavelet propagated with reflection on the ground.

Fig. 4. The two possible types of propagated wavelets.

\section{Propagation over a planar ground with a constant surface impedance $(W W P-M)$}

If a planar ground with a constant surface impedance is assumed at $z=0$, the ground reflections could be included in the propagation matrix, so that the method is denoted by WWP-M.

In WWP-M, both the ground reflections and the apodization at the top boundary are included in $\tilde{\mathbf{M}}$, which is expressed by

$$
\tilde{\mathbf{M}}_{(\cdot, \cdot),\left(l^{\prime}, p^{\prime}\right)}=\mathbf{H}^{\mathrm{c}} \mathbf{M}_{(\cdot, \cdot),\left(l^{\prime}, p^{\prime}\right)}^{\mathrm{g}},
$$

with $\mathbf{M}^{\mathrm{g}}$ the propagation matrix considering the reflections over the ground.

In order to fill $\mathbf{M}^{\mathrm{g}}$, for each wavelet $\chi_{l^{\prime}, p^{\prime}}(0, \cdot)$, two cases may occur, depending if the propagated wavelet $\chi_{l^{\prime}, p^{\prime}}(\Delta x, \cdot)$ is reflected over the ground. A threshold of $-50 \mathrm{~dB}$ below the maximum value is introduced to assess the limits of the wavelet vertical extension. The wavelet is reflected by the ground, if the limits reaches the ground after propagation on $\Delta x$.

- If $\chi_{l^{\prime}, p^{\prime}}(\Delta x, \cdot)$ does not reach the ground, as illustrated in Fig. 4a, $\mathbf{M}_{(\cdot, \cdot)}^{\mathrm{g}}\left(l^{\prime}, p^{\prime}\right)$ is filled by the same way as presented in Section III-B1. The wavelets propagated in free space satisfy the same shift-invariant property as the wavelets before propagation.

- If $\chi_{l^{\prime}, p^{\prime}}(\Delta x, \cdot)$ is reflected by the ground, as in Fig. $4 \mathrm{~b}$, it is simulated again by DSSF in the presence of the ground reflection. Then, the corresponding column of $\mathbf{M}^{\mathrm{g}}$ is filled. Indeed, the shift-invariance is lost in this case and the steps of Fig. 3 must be applied for each of these wavelets separately.
By replacing $\tilde{\mathbf{M}}$ in (8) by (15), the wave propagation over a planar ground with constant surface impedance can be efficiently simulated by WWP-M.

\section{Propagation OVER A NON CONSTANT IMPEDANCE GROUND AND/OR WITH AN IRREGULAR RELIEF (WWP-H)}

In SSW, a dielectric ground is accounted for by a local image technique with a Dirichlet condition after a change of variable as proposed by Dockery and Kuttler [27]. Since the discrete wavelets we use are not symmetric, the local image method cannot be applied on the wavelets directly.

In the case with a relief, the classical staircase modelling [5] is applied in the space domain. Therefore, in any of these cases, the lower part of the field is propagated with SSW to consider the boundary condition and the relief. This requires an hybridization between WWP and SSW, denoted hereafter as WWP-H.

This method is presented with a planar impedance ground for the sake of simplicity. Then, the implementation for an irregular relief is added to the method.

\section{A. Propagation over a planar ground with a variable surface impedance}

The hybridization method WWP-H combines the WWP method with the concept of DMFT [27] and the local image source method of SSW [22]. Here, $w$ is the variable change of the field $u$ as in DMFT [27]. The wavelet coefficients of $w$ are denoted as $W_{(l, p)}$. The main steps of the method are illustrated in Fig. 5 and introduced as follows:

1) As illustrated by the green frame in Fig. 5(a), we focus on the wavelet coefficients $W_{(l, p)}$ in a thin layer for $z(l, p) \in\left[-z_{\text {im }}, 2 z_{\text {im }}\right]$, with $z_{\text {im }}=N_{\text {im }} \Delta z$. The value of $2 z_{\mathrm{im}}$ is assumed to be larger than the width of the largest wavelet and its field spread with propagation. In practice, $N_{\mathrm{im}} \ll N_{\mathrm{z}}$.

2) Applying inverse FWT on these coefficients, the corresponding field is obtained, denoted as $w_{p_{z}}^{(1)}$, with $p_{z} \in\left[-N_{\mathrm{im}}, 2 N_{\mathrm{im}}\right]$, illustrated in Fig. 5(b).

Due to the truncation of the coefficients, the field $w_{p_{z}}^{(1)}$ has spurious oscillations near the bottom and top limits, i.e., at $p_{z}=-N_{\mathrm{im}}$ and $p_{z}=2 N_{\mathrm{im}}$. The choice of $N_{\mathrm{im}}$ guarantees that after one step $\Delta x$, this error does not reach the domain $p_{z} \in\left[0, N_{\mathrm{im}}\right]$, which is illustrated by the red line in Fig. 5(b).

3) The field in the local image layer (with $p_{z} \in\left[-N_{\mathrm{im}}, 0\right]$ ) is updated by the upper part (with $p_{z} \in\left[0, N_{\mathrm{im}}\right]$ ), as illustrated by the red line in Fig. 5(c). The field $w^{(2)}$ is defined as

$$
w_{p_{z}}^{(2)}= \begin{cases}w_{p_{z}}^{(1)} & \text { for } p_{z} \in\left[1,2 N_{\mathrm{im}}\right], \\ 0 & \text { for } p_{z}=0, \\ -w_{-p_{z}}^{(1)} & \text { for } p_{z} \in\left[-N_{\mathrm{im}},-1\right],\end{cases}
$$

The spurious oscillation at the bottom limit in Fig. 5(b) is cleared up in this step. The error at the top limit is addressed in the next step.

4) The coefficients $W_{(l, p)}^{(2)}$, with $z(l, p) \in\left[-z_{\mathrm{im}}, 2 z_{\mathrm{im}}\right]$, is obtained by applying FWT on $w^{(2)}$. These coefficients 
are represented by the yellow grid in Fig. 5 (d). The coefficients with $z(l, p) \in\left[-z_{\mathrm{im}}, z_{\mathrm{im}}\right]$ are needed for the next step, illustrated by the blue frame in Fig. 5 (d). The upper part with $\left.z(l, p) \in] z_{\mathrm{im}}, 2 z_{\mathrm{im}}\right]$ is dropped off to remove the error at the top limit.

5) The final coefficients $W^{g}$ are obtained by

$$
W_{(l, p)}^{\mathrm{g}}= \begin{cases}W_{(l, p)}^{(2)} & \text { if } z(l, p) \in\left[-z_{\mathrm{im}}, z_{\mathrm{im}}\right] \\ W_{(l, p)} & \text { if } \left.z(l, p) \in] z_{\mathrm{im}}, z_{\max }\right]\end{cases}
$$

As illustrated in Fig. 5 (e), $W^{\mathrm{g}}$ are the combination of the coefficients inside the pink frame of (a) and the ones inside the blue frame of (d).

With the WWP-H method, the wavelets are not required to be symmetric. This method avoids the spurious errors and wavelet transforms are performed only on a thin layer. The operator for applying this method is denoted as $\mathbf{G}$. The propagation on $\Delta x$ is computed by

$$
W_{p_{x}+1}=\mathbf{R}^{\mathrm{c}} \tilde{\mathbf{M G}} \mathbf{C} W_{p_{x}} .
$$

Finally, the field at the final step $u_{N_{x}}$ is calculated by recursions introduced in DMFT [27] from $w_{N_{x}}$, which is obtain by applying inverse FWT on $W_{N_{x}}$.

\section{B. Propagation over an irregular relief}

The WWP-H can account for an irregular relief. The staircases terrain model [5] is used. At each range step $p_{x} \Delta x$, the height of the ground is $z_{p_{x}}^{\mathrm{g}}$. The irregular relief is accounted by adapting $+z_{p_{x}}^{\mathrm{g}}$ in the equations of Section IV-A. Other terrain models, such as the shift map terrain model, can also be incorporated in WWP-H. However, at each range step, the propagators should be calculated again. This can be realized with a negligible calculation cost by using a fast calculation of the propagators, as proposed in [28].

As a conclusion, the hybridization method WWP-H can model the propagation over an impedance ground and/or with an irregular relief. In the next section, the computational complexities of the propagation methods WWP-M, WWP-H, SSW, and DSSF are compared.

\section{COMPLEXITIES OF DIFFERENT PROPAGATION METHODS}

Each propagation method includes two parts, the wavelet/Fourier transform part and the propagation part. The computational complexities of both parts are compared.

1) In DSSF, the complexity of FFT is $O\left(N_{z} \log N_{z}\right)$ and the one for propagation is $O\left(N_{z}\right)$ [27].

2) In SSW, the complexity of FWT is $O\left(N_{z}+N_{\mathrm{im}}\right) \approx$ $O\left(N_{z}\right)$. The complexity of the propagation is $O\left(N_{e}\right)$ where $N_{e} \leq N_{M} N_{s}$. Here, $N_{M}, N_{s}$ are the numbers of the non-zero elements of $\mathbf{M}$ and $U$, respectively. Therefore, $N_{M}$ and $N_{s}$ are much smaller than $N_{z}$ due to the high compression ratio of the wavelet decomposition. [22]

3) In WWP-M, as introduced in Section III, no wavelet transform is performed. For the propagation, the complexity is the same as in SSW, which is $O\left(N_{e}\right)$.
TABLE I

COMPUTATIONAL COMPLEXITIES OF WWP, SSW AND DSSF FOR THE PROPAGATION ON $\Delta x$

\begin{tabular}{l|l|l|l|l}
\hline & DSSF & SSW & WWP-M & WWP-H \\
\hline Tran. & $O\left(N_{z} \log N_{z}\right)$ & $O\left((1+\beta) N_{z}\right)$ & -- & $O\left(3 \beta N_{z}\right)$ \\
\hline Prop. & $O\left(N_{z}\right)$ & $O\left(N_{e}\right)$ & $O\left(N_{e}\right)$ & $O\left(N_{e}\right)$ \\
\hline
\end{tabular}

4) In WWP-H, as introduced in Section IV, a thin layer of the wavelet coefficients near the ground is transformed in the field domain. The complexity of this transform is $O\left(3 N_{\mathrm{im}}\right)=O\left(3 \beta N_{z}\right)$ with $\beta \ll 1$. For the propagation, the complexity is $O\left(N_{e}\right)$.

The computational complexities of DSSF, SSW, WWP-M, and WWP-H are summarized in Table I. The complexities of the propagation part (prop.) and the transform part (tran.) are compared. For the propagation part, WWP-M, WWP-H, and SSW are the same. In practice, their complexities are much smaller than DSSF, since $N_{M} \ll N_{z}, N_{s} \ll N_{z}$ due to the compression and $N_{e} \leq N_{M} N_{s}$. For the transform part, WWP$\mathrm{M}$ is obviously the best and the complexity of WWP-H is smaller than SSW. They are both faster than DSSF.

As a conclusion, the complexities of WWP-M and WWP-H are less than SSW, and they are both much lower than DSSF. Both methods WWP-M and WWP-H are applied with different ground or relief conditions in the next section.

\section{NUMERICAL TESTS}

Various simulations are performed to test the wavelet-towavelet propagation methods. Results accuracies are compared to analytic solutions, the geometrical optics method, DSSF, and SSW. Moreover, the computational efficiencies are compared.

Due to the compression threshold on the propagation matrix $\mathrm{M}, V_{\mathrm{M}}$ and on the signal, $V_{\mathrm{s}}$. Numerical errors exist compared to the analytic solution. According to the numerical experiments in [29], the expected error $\delta_{N_{x}} \lesssim V_{\mathrm{P}} N_{x}+V_{\mathrm{s}} N_{x}$, with $N_{x}$ the number of range steps. Thus, the error increases with the total number of iterations.

\section{A. Propagation in an atmosphere without ground}

Firstly, propagations in a homogeneous atmosphere without ground are tested. The objective is to show the accuracy and efficiency of WWP. A complex source point (CSP) [30] is chosen as source. Its propagation in vacuum can be analytically calculated and serves here as a reference. The parameters of the CSP are: frequency $f=3 \mathrm{GHz}, z_{\mathrm{w} 0}=-50 \mathrm{~m}, y_{\mathrm{s}}=0 \mathrm{~m}$, $z_{\mathrm{s}}=300 \mathrm{~m}$, and $W_{0}=3 \mathrm{~m}$.

The simulation parameters are $x_{0}=0 \mathrm{~m}, x_{\max }=5 \mathrm{~km}$, $\Delta x=10 \mathrm{~m}, z_{\max }=600 \mathrm{~m}$, and $\Delta z=0.2 \mathrm{~m}$. Thus, $N_{x}=500$ and $N_{z}=3000$. Besides, absorbing windows are applied on the domains $z \in[0,200] \mathrm{m}$ and $z \in[400,600] \mathrm{m}$.

As a preliminary test, we choose the wavelet parameters the same as in [22]. The chosen orthonormal wavelet basis is sym6, with the maximum decomposition level $L=3$.

WWP and SSW with compressions on both $\mathbf{M}$ and the wave are applied. The chosen thresholds are $V_{\mathrm{P}}=2 \times 10^{-5}$, 


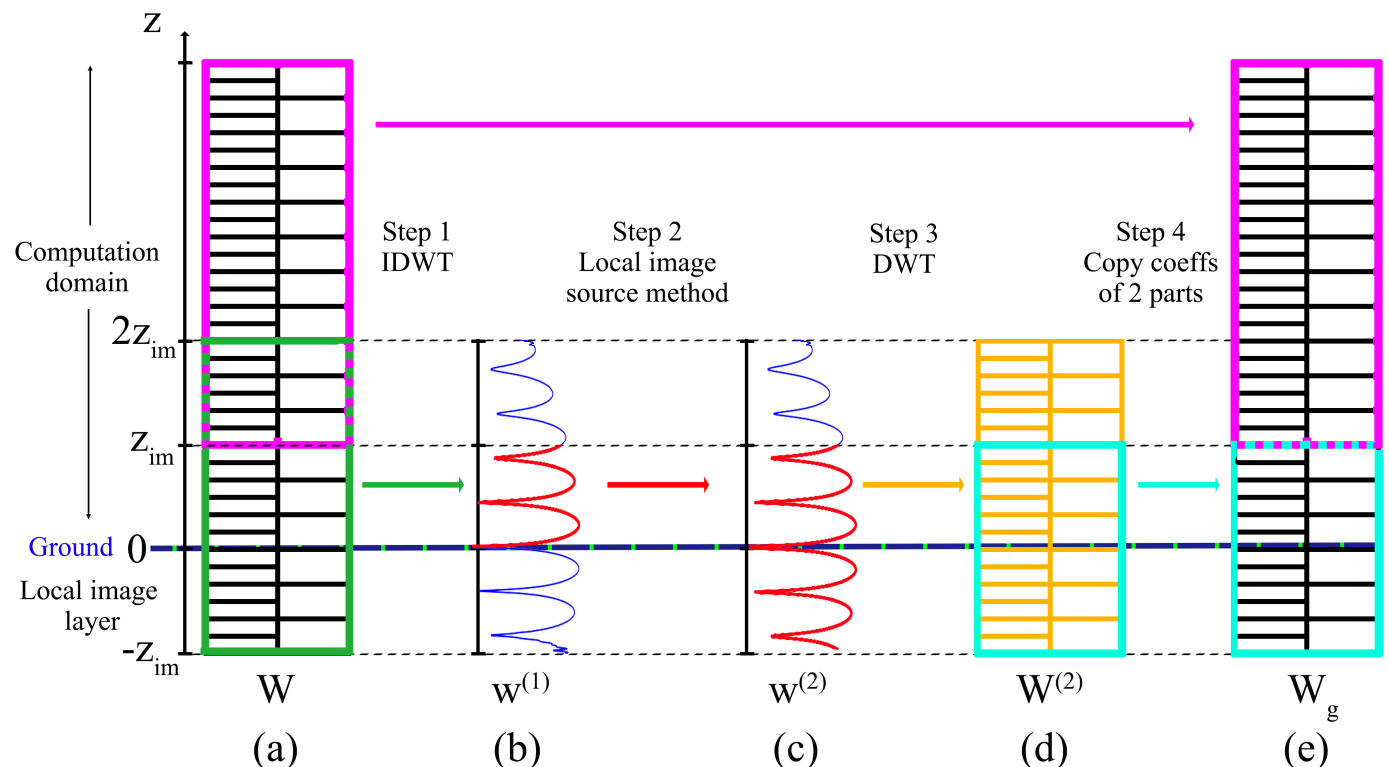

(a)

(b)

(c)

(d)

(e)

Fig. 5. Illustration of the hybridization method of WWP with the local image source method in SSW (WWP-H).

$V_{\mathrm{s}}=2 \times 10^{-3}$. Besides, $V_{\mathrm{P}}$ is normalized by the maximum amplitude of the dilated wavelets, and $V_{\mathrm{s}}$ is normalized by the maximum amplitude of the fields for each step in distance. Since $N_{x}=500$, according to the discussion about the error evaluation with the thresholds values and the iterations number, the expected error is $-37.8 \mathrm{~dB}$.

The propagation of the electric field using WWP is plotted in Fig. 6a. The normalized final electrical fields of WWP, SSW and the analytic solution are plotted in Fig. 6b. The differences between the three methods are quite small. RMS differences and computation times are listed in Table III. The RMS difference of WWP with analytic solution meets with the expected value $-37.8 \mathrm{~dB}$. The computation time of WWP is 3 times shorter than SSW and 10 times shorter than DSSF. Besides, the time for filling $\mathrm{M}$ is $0.7 \mathrm{~s}$. The propagation of a CSP in a homogeneous atmosphere using WWP is successfully tested with a high efficiency and a good accuracy.

In order to evaluate the errors and the computation times with different wavelet parameters, we test different combinations of wavelet family and multiscale level, see Table II. The case with sym 6 and $L=2$ has the highest simulation accuracy and computational efficiency. For the wavelet families sym 4 and sym2, the cases with $L=2$ and $L=3$ respectively have a higher accuracy than the others. Because the test can be regarded as the propagation in free-space, the errors are due to the compressions applied on the matrix and the signal. These error levels can be estimated as introduced in [26].

In order to validate the method of apodization, as introduced in III-B2, the simulation range is extended to $10 \mathrm{~km}$ (as plotted in Fig.7) and tested with different wavelet parameters are performed. Different decomposition levels $L$ and wavelet families are chosen, the results are listed in Table IV. The errors remain small and acceptable. The best parameters are with sym 6 and $L=2$. The result is quite similar to the propagation on $5 \mathrm{~km}$. It is believed the errors are mainly due
TABLE II

TESTS USING WWP WITH DIFFERENT WAVELET PARAMETERS.

\begin{tabular}{c||c|c|c|c|c}
\hline Wavelet parameters & $\begin{array}{c}\mathrm{L}=1 \\
\text { sym6 }\end{array}$ & $\begin{array}{c}\mathrm{L}=2 \\
\text { sym6 }\end{array}$ & $\begin{array}{c}\mathrm{L}=3 \\
\text { sym6 }\end{array}$ & $\begin{array}{c}\mathrm{L}=4 \\
\text { sym6 }\end{array}$ & $\begin{array}{c}\mathrm{L}=5 \\
\text { sym6 }\end{array}$ \\
\hline RMS diff. of ampl. (dB) & -50.7 & -52.4 & -35.6 & -34.9 & -31.8 \\
\hline Time (s) & 0.28 & 0.27 & 0.29 & 0.31 & 0.30 \\
\hline \hline Wavelet parameters & $\begin{array}{c}\mathrm{L}=1 \\
\text { sym4 }\end{array}$ & $\begin{array}{c}\mathrm{L}=2 \\
\text { sym4 }\end{array}$ & $\begin{array}{c}\mathrm{L}=3 \\
\text { sym4 }\end{array}$ & $\begin{array}{c}\mathrm{L}=4 \\
\text { sym4 } 4\end{array}$ & $\begin{array}{c}\mathrm{L}=5 \\
\text { sym4 }\end{array}$ \\
\hline RMS diff. of ampl. (dB) & -38.1 & -42.2 & -38.0 & -33.8 & -31.7 \\
\hline Time (s) & 0.31 & 0.31 & 0.31 & 0.33 & 0.32 \\
\hline \hline Wavelet parameters & $\mathrm{L}=1$ & $\mathrm{~L}=2$ & $\mathrm{~L}=3$ & $\mathrm{~L}=4$ & $\mathrm{~L}=5$ \\
sym2 & sym2 & sym2 & sym2 & sym2 \\
\hline RMS diff. of ampl. (dB) & -42.3 & -36.8 & -38.1 & -34.6 & -33.7 \\
\hline Time (s) & 0.41 & 0.41 & 0.41 & 0.45 & 0.46 \\
\hline
\end{tabular}

TABLE III

COMPUTATION TIMES AND RMS DIFFERENCES OF AMPLITUDE OF WWP, SSW, DSSF TO THE ANALYTIC SOLUTION.

\begin{tabular}{c||c|c|c}
\hline Methods & WWP & SSW & DSSF \\
\hline RMS difference of amplitude (dB) & -35.6 & -36.9 & -103.3 \\
\hline Time (s) & 0.5 & 1.5 & 4.9 \\
\hline \multicolumn{2}{|c}{}
\end{tabular}

TABLE IV

TESTS USING WWP WITH DIFFERENT WAVELET PARAMETERS.

\begin{tabular}{c|c|c|c|c|c}
\hline Wavelet parameters & $\begin{array}{c}\mathrm{L}=1 \\
\text { sym6 }\end{array}$ & $\begin{array}{c}\mathrm{L}=2 \\
\text { sym6 }\end{array}$ & $\begin{array}{c}\mathrm{L}=3 \\
\text { sym6 }\end{array}$ & $\begin{array}{c}\mathrm{L}=4 \\
\text { sym6 }\end{array}$ & $\begin{array}{c}\mathrm{L}=5 \\
\text { sym6 }\end{array}$ \\
\hline RMS diff. of ampl. (dB) & -49.3 & -67.6 & -33.7 & -32.0 & -31.4 \\
\hline Time (s) & 0.61 & 0.60 & 0.61 & 0.65 & 0.63 \\
\hline Wavelet parameters & $\mathrm{L}=1$ & $\mathrm{~L}=2$ & $\mathrm{~L}=3$ & $\mathrm{~L}=4$ & $\mathrm{~L}=5$ \\
& sym4 & sym4 & sym4 & sym4 & sym4 \\
\hline RMS diff. of ampl. (dB) & -32.3 & -45.9 & -53.7 & -37.0 & -33.0 \\
\hline Time (s) & 0.76 & 0.61 & 0.63 & 0.63 & 0.65 \\
\hline \hline Wavelet parameters & $\mathrm{L}=1$ & $\mathrm{~L}=2$ & $\mathrm{~L}=3$ & $\mathrm{~L}=4$ & $\mathrm{~L}=5$ \\
& sym2 & sym2 & sym2 & sym2 & sym2 \\
\hline RMS diff. of ampl. (dB) & -37.9 & -31.8 & -50.24 & -37.3 & -35.7 \\
\hline Time (s) & 0.93 & 0.90 & 0.91 & 0.92 & 0.91 \\
\hline
\end{tabular}

to the compressions, but not from the apodization. The errors caused by the apodization can be neglected. 


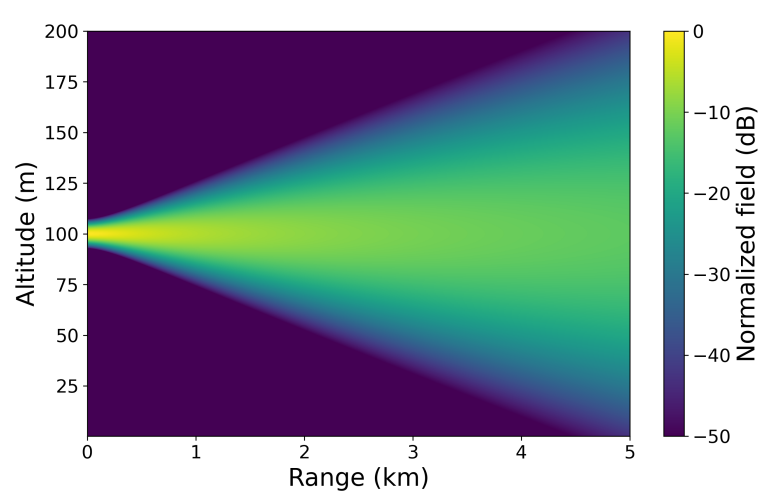

(a) Propagation using WWP.

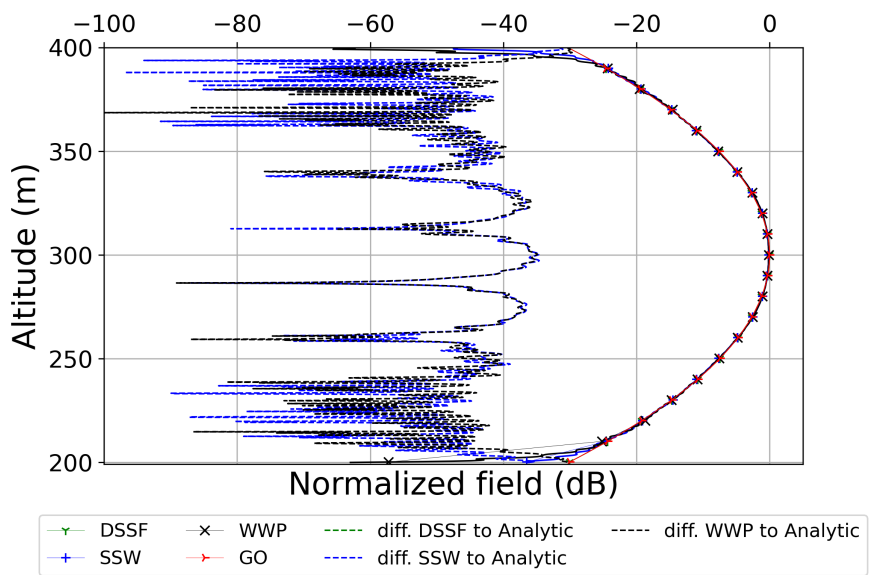

(b) Normalized electric fields at the final step obtained by WWP, SSW, DSSF, and the analytic solution (full lines) and differences of WWP-M, SSW, and DSSF to the analytic solution (dashed lines).

Fig. 6. Propagation in a homogeneous atmosphere of a CSP $(5 \mathrm{~km})$ with $L=3$ and wavelet basis sym6.

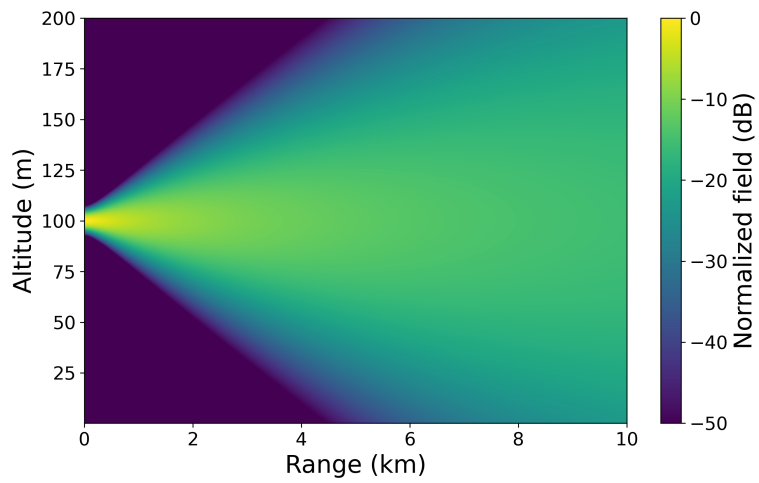

Fig. 7. Propagation in a homogeneous atmosphere of a CSP $(10 \mathrm{~km})$ with $L=3$ and wavelet basis sym6.
TABLE V

TIMES AND RMS DIFFERENCES OF WWP-M, SSW, DSSF COMPARED TO GO FOR PROPAGATION OVER A PLANAR GROUND OF CSP.

\begin{tabular}{l||c|c|c}
\hline Methods & WWP-M & SSW & DSSF \\
\hline $\begin{array}{l}\text { RMS difference of amplitude } \\
\text { at } 10 \mathrm{~km}(\mathrm{~dB})\end{array}$ & -27.9 & -27.9 & -27.9 \\
\hline $\begin{array}{l}\text { RMS difference of amplitude } \\
\text { at } 20 \mathrm{~km}(\mathrm{~dB})\end{array}$ & -36.2 & -36.2 & -36.4 \\
\hline $\begin{array}{l}\text { RMS difference of amplitude } \\
\text { at } 50 \mathrm{~km}(\mathrm{~dB})\end{array}$ & -50.6 & -50.1 & -89.0 \\
\hline Total computation time (s) & 0.5 & 6.1 & 10.2 \\
\hline
\end{tabular}

\section{B. Propagation over a planar ground with a constant surface impedance}

The propagation over a planar ground with a constant surface impedance in a homogeneous atmosphere is considered. A CSP with $W_{0}=1 \mathrm{~m}$ and $h_{\mathrm{s}}=5 \mathrm{~m}$ is considered.

The simulation parameters are $x_{0}=0 \mathrm{~m}, x_{\max }=50 \mathrm{~km}$, $\Delta x=100 \mathrm{~m}, z_{\max }=819.2 \mathrm{~m}, \Delta z=0.2 \mathrm{~m}$. Thus, $N_{x}=500$. The compression thresholds are the same as in Test VI-A. The expected error is $-37.8 \mathrm{~dB}$.

In this test, the wavelet sym6 with $L=2$ is applied. The characteristics of the impedance ground are $\epsilon_{r}=20$ and $\sigma=$ $0.1 \mathrm{~S} / \mathrm{m}$.

1) Propagation in a homogeneous medium: The WWP-M method introduced in Section III-D is compared to SSW, DSSF and GO to test its accuracy.

The propagation using WWP-M is shown in Fig. 8a. The interferences of the direct and reflected waves are well simulated. The normalized final electrical fields at the final step of the different methods are plotted by solid lines in Fig. 8d. The differences of SSW, WWP-M, and DSSF to GO are illustrated by the dotted lines. The results of WWP-M meets to the expectation. Both methods are very accurate. In addition, the normalized final electrical fields at $10 \mathrm{~km}$ and $20 \mathrm{~km}$ are plotted in Fig. 8b and 8c, respectively.

The simulation results including RMS differences and computation times are shown in Table V. The computation time of the WWP-M method is 6.6 times shorter than SSW and 16.2 times shorter than DSSF. The time of creating the propagation matrix $\mathrm{M}$ is $2.5 \mathrm{~s}$. In total, 548 propagations of near-ground wavelets (Fig. 4b) are performed.

2) Propagation with an atmospheric duct: In the vertical direction, we consider a surface-based duct, which is modeled by a trilinear modified refractivity, as illustrated in Fig. 9. The parameters are: $M_{0}=330$ M-units, $z_{b}=50 \mathrm{~m}, z_{t}=100 \mathrm{~m}$, with gradients $c_{0}=0.118 \mathrm{M}$-units $/ \mathrm{m}, c_{2}=-0.3 \mathrm{M}$-units $/ \mathrm{m}$.

In order to test the phase screen method applied on the wavelet coefficients presented in Section III-C, tests with different wavelet parameters are performed. The simulation results are listed in Table VI. As a comparison, the computational time of SSW with sym6 and $L=3$ (the same parameter as in [22]) is $5.85 \mathrm{~s}$. Under this condition, the RMS difference of SSW to DSSF is $-45.2 \mathrm{~dB}$. The computational time of DSSF is $8.97 \mathrm{~s}$.

According to the results in Table VI, several conclusions can be drawn. First, in all cases of WWP-M, the computation times are much faster than SSW and DSSF. For example, the 


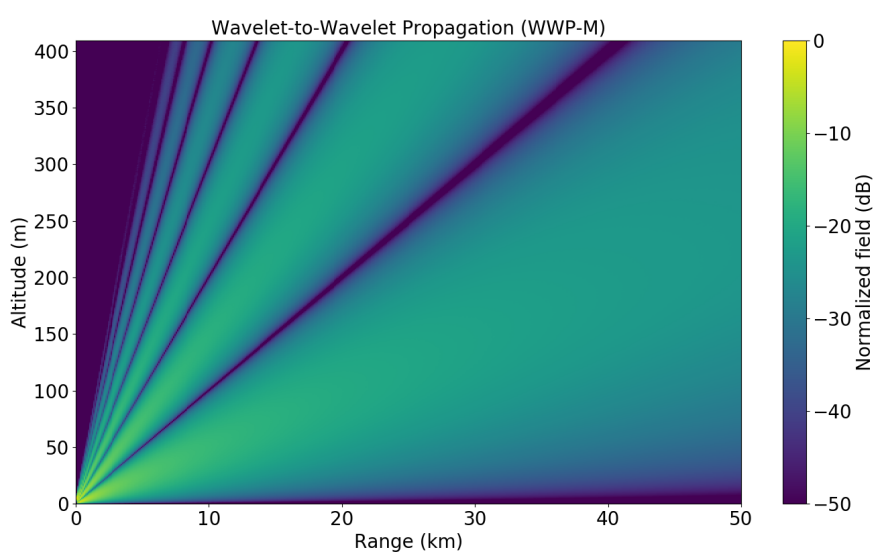

(a) Propagation using WWP-M.

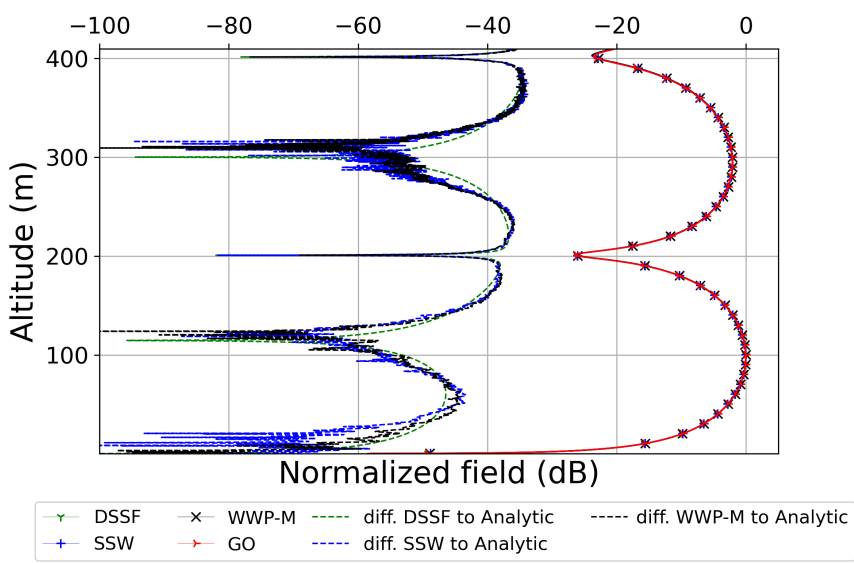

(c) Normalized electric fields at $20 \mathrm{~km}$.

Fig. 8. Propagation over an impedance planar ground of CSP.

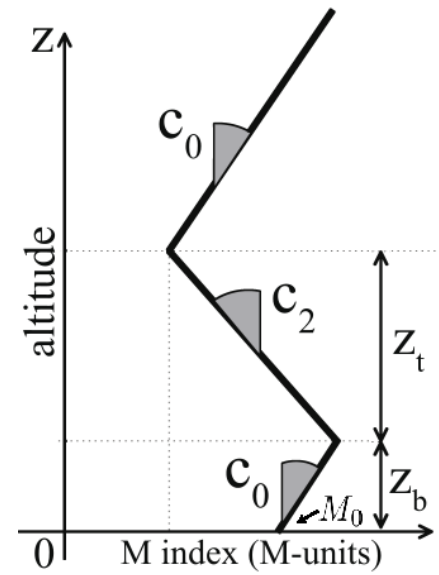

Fig. 9. Trilinear model of refractivity

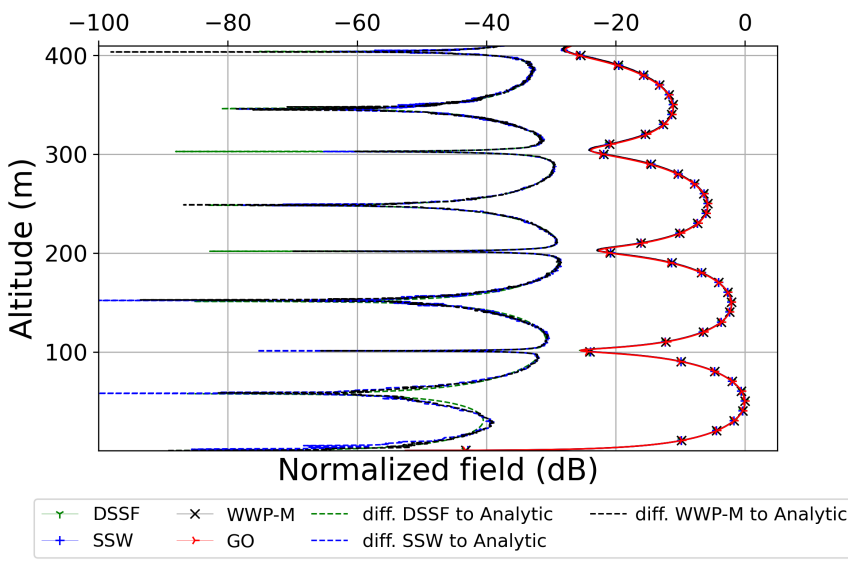

(b) Normalized electric fields at $10 \mathrm{~km}$ obtained by WWP-M, SSW, and DSSF (full lines) and differences of WWP-M and SSW to DSSF (dashed lines).

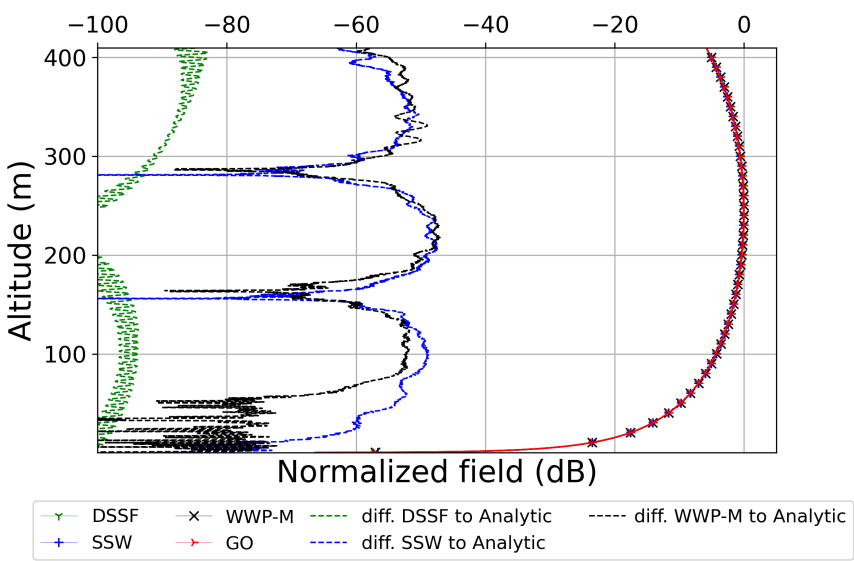

(d) Normalized electric fields at $50 \mathrm{~km}$.

case with sym6 and $L=2$ is about 9 times faster than SSW and 14 times faster than DSSF. Second, the RMS differences of amplitude between WWP-M and DSSF are greater. This is because in WWP-M, the refractivity is applied on the wavelet coefficient directly. This accounts for considering a constant refractive index on all the support of the wavelet. The error remains small when the most dilated wavelets are of narrow width. That is to say, a smaller wavelet order and a smaller maximum decomposition level $L$ can achieve a better accuracy. This theoretical analysis is tested and the simulation results with different wavelet parameters are listed in Table VI. In cases with small wavelet order and small $L$, the accuracy with WWP-M is just a little worse than SSW. For example, in the case with sym 2 and $L=1$ (plotted in Fig. 10a and Fig. 10b), the RMS difference of WWP-M to DSSF is $-40.8 \mathrm{~dB}$ and the RMS difference of SSW to DSSF is $-45.2 \mathrm{~dB}$. The difference of simulation accuracy is small, but the computation time of WWP-M is about 5 times faster than 
TABLE VI

TESTS USING WWP-M WITH DIFFERENT WAVELET PARAMETERS.

\begin{tabular}{c||c|c|c|c|c}
\hline Wavelet parameters & $\begin{array}{c}\mathrm{L}=1 \\
\text { sym6 }\end{array}$ & $\begin{array}{c}\mathrm{L}=2 \\
\text { sym6 }\end{array}$ & $\begin{array}{c}\mathrm{L}=3 \\
\text { sym6 }\end{array}$ & $\begin{array}{c}\mathrm{L}=4 \\
\text { sym6 }\end{array}$ & $\begin{array}{c}\mathrm{L}=5 \\
\text { sym6 }\end{array}$ \\
\hline RMS diff. of ampl. (dB) & -37.7 & -27.6 & -20.1 & -13.4 & -5.9 \\
\hline Time (s) & 0.78 & 0.65 & 0.76 & 0.81 & 0.97 \\
\hline \hline Wavelet parameters & $\begin{array}{c}\mathrm{L}=1 \\
\text { sym4 }\end{array}$ & $\begin{array}{c}\mathrm{L}=2 \\
\text { sym4 }\end{array}$ & $\begin{array}{c}\mathrm{L}=3 \\
\text { sym4 }\end{array}$ & $\begin{array}{c}\mathrm{L}=4 \\
\text { sym4 }\end{array}$ & $\begin{array}{c}\mathrm{L}=5 \\
\text { sym4 } 4\end{array}$ \\
\hline RMS diff. of ampl. (dB) & -35.3 & -26.3 & -18.9 & -12.3 & -6.7 \\
\hline Time (s) & 1.01 & 0.95 & 1.01 & 1.09 & 1.30 \\
\hline \hline Wavelet parameters & $\mathrm{L}=1$ & $\mathrm{~L}=2$ & $\mathrm{~L}=3$ & $\mathrm{~L}=4$ & $\mathrm{~L}=5$ \\
sym2 & sym2 & sym2 & sym2 & sym2 \\
\hline RMS diff. of ampl. (dB) & -40.8 & -34.3 & -28.1 & -22.0 & -17.1 \\
\hline Time (s) & 1.86 & 1.82 & 1.77 & 1.82 & 1.92 \\
\hline
\end{tabular}

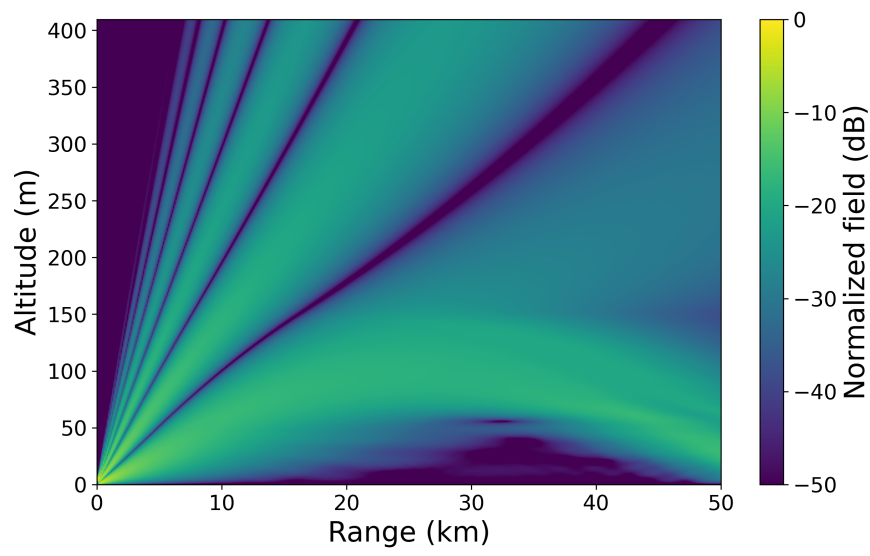

(a) Propagation using WWP-M with sym 2 and $L=1$.

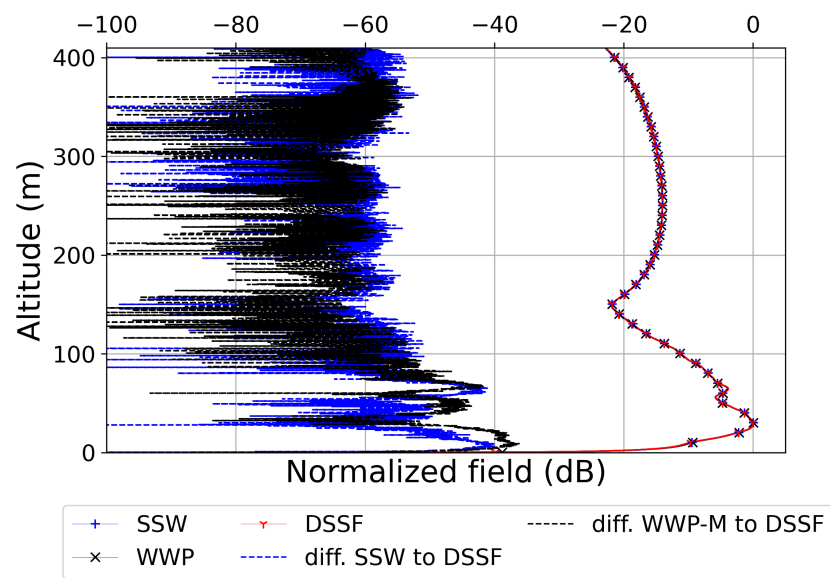

(b) Normalized electric fields at the final step obtained by WWP-M with sym2 and $L=1$, SSW, and DSSF (full lines) and differences of WWP-M and SSW to DSSF (dashed lines).

Fig. 10. Propagation over an impedance planar ground of CSP.

SSW. So, the advantage of WWP-M is clear in computational efficiency while keeping a good accuracy.

\section{Long-range propagation in a complex environment}

In this part, we test a long-range propagation over an irregular relief in an inhomogeneous atmosphere.

A surface-based duct is considered the same as in Test VI-B2. The relief is chosen as 1 triangular hill of height $80 \mathrm{~m}$ with a support of $40 \mathrm{~m}$ and 1 Gaussian hill of height
TABLE VII

TIMES AND RMS DIFFERENCES OF WWP-H AND SSW TO DSSF FOR LONG-RANGE PROPAGATION OF CSP OVER AN IRREGULAR RELIEF.

\begin{tabular}{c||c|c|c}
\hline Methods & WWP-H & SSW & DSSF \\
\hline RMS difference of amplitude (dB) & -38.8 & -50.1 & \\
\hline Time (s) & 3.1 & 9.4 & 13.5 \\
\hline
\end{tabular}

$80 \mathrm{~m}$ with $w_{0}=10 \mathrm{~km}$. The characteristics of the impedance ground are $\epsilon_{r}=20$ and $\sigma=0.1 \mathrm{~S} / \mathrm{m}$. The propagation range is $x_{\max }=100 \mathrm{~km}$. The range step is $200 \mathrm{~m}, N_{x}=500$. We choose $V_{\mathrm{P}}=2 \times 10^{-5}$ and $V_{\mathrm{s}}=2 \times 10^{-3}$. The wavelet parameter is set by sym 2 and $L=1$.

The WWP-H method is used. The number of points in the local image layer is chosen $N_{\mathrm{im}}=200$ (with $N_{z}=4096$ ).

The propagation using WWP-H in the vertical plane is plotted in Fig. 11a. The refractive effects of the surface-based duct and the reflection by the irregular ground are properly simulated. The final fields and the differences of WWP-H and SSW to DSSF are plotted in Fig. 11b. In addition, the comparison of range variation at the height $150 \mathrm{~m}$ is plotted in Fig. 11c. The error between WWP-H and DSSF remains small along the range.

The simulation results including RMS differences and computation times are given in Table VII. The difference of WWP$\mathrm{H}$ to DSSF is $-38.8 \mathrm{~dB}$, which well satisfies the simulation requirement. The computation time of WWP-H is $3.1 \mathrm{~s}$, which is about 3 times faster than SSW and 4 times faster than DSSF. Therefore, WWP-H has a better computational efficiency while keeping a very good efficiency, even on complex scenarios.

\section{Guideline for choosing the wavelet parameters}

In [29], a theoretical closed-form formula for the accumulated compression error is given. Both the signal compression error and matrix compression error accumulate with $N_{x}$. The signal compression error after $N_{x}$ horizontal iterations fulfils $\delta_{N_{x}}^{s} \lesssim N_{x} V_{s}$ and the matrix compression error fulfils $\delta_{N_{x}}^{m} \lesssim N_{x} V_{M}$, with $V_{\mathrm{M}}$ the threshold value on the matrix, $V_{\mathrm{s}}$ the threshold value on the signal, and $N_{x}$ the number of range steps. The modellings of ground condition, irregular terrain, and phase screen are the same in SSW and DSSF. So, the error is believed to be entirely caused by the thresholdings.

In WWP-M and WWP-H, the best choice of wavelet parameters not only depends on $N_{z}$, but also the models of phase-screen, apodization, and the hybridisation method for considering a variable impedance ground and/or irregular relief. In general, it is hard to determine the best choice for all cases. However, the numerical simulations show that the computation time is not so dependent on the wavelet parameters. Thus, for a practical complex scenario assuming a varying atmosphere, any wavelet choice implying a small support is reasonable. Our main recommendation is to keep $L \leq 2$ to keep this support small.

\section{CONCLUSION}

In this paper, the wavelet-to-wavelet propagation method has been presented, as an improvement to the split-step wavelet 


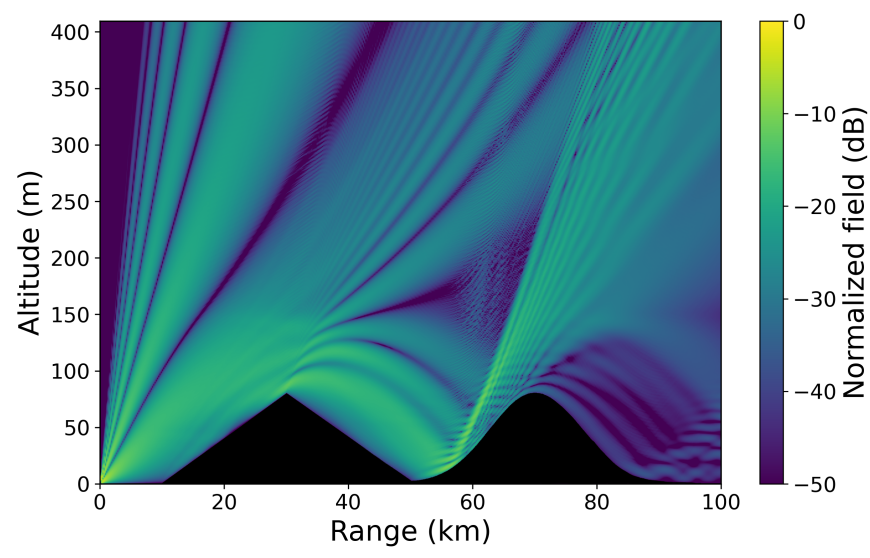

(a) Propagation using WWP-H.

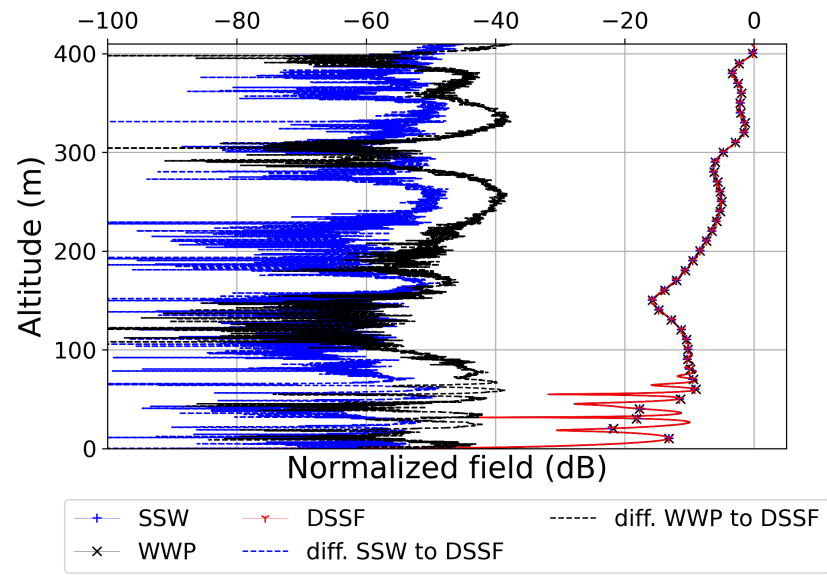

(b) Normalized electric fields at the final step obtained by WWP-H, SSW, and DSSF (full lines) and differences of WWP-H and SSW to DSSF (dashed lines).

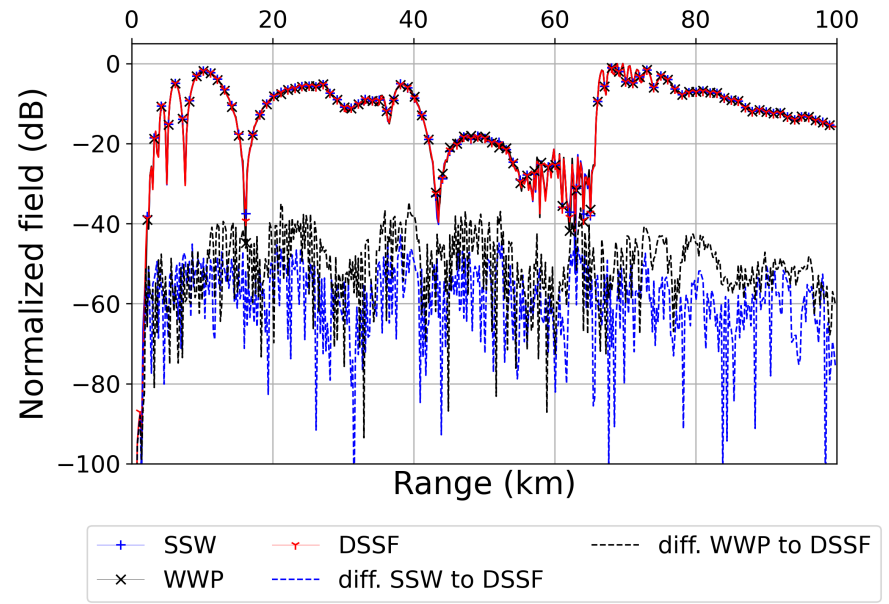

(c) Normalized electric fields along $+x$ direction at 150 m obtained by WWP$\mathrm{H}$, SSW, and DSSF (full lines) and differences of WWP-H and SSW to DSSF (dashed lines).

Fig. 11. Propagation of CSP over an impedance ground with an irregular relief. method. The method is shown to be fast and accurate on planar ground. To account for relief, the hybridization with SSW has been presented and successfully tested with numerical experiments. The computational complexity is smaller than other methods. In the tests, this method has well simulated the long-range propagation in a complex environment. The wavelet-to-wavelet methods have shown a very good efficiency compared to SSW and DSSF. Moreover, a very good accuracy is also achieved. To continue this research, several future works could be considered. The method of WWP could be extended to solve three dimension problems, a more accurate model of the phase-screen and the link between the wavelet support and error at the phase screen step should be further investigated.

\section{ACKNOWLEDGEMENTS}

This work was supported by Science Foundation of Tianjin Municipal Education Commission (grant number: 2020KJ037) and it has been supported during Hang Zhou's Ph.D. thesis research by ENAC.

\section{REFERENCES}

[1] M. A. Leontovich and V. A. Fock, "Solution of propagation of electromagnetic waves along Earth's surface by the method of parabolic equations," J. Phys. USSR, vol. 10, pp. 13-23, 1946.

[2] A. V. Popov, "Solution of a parabolic equation of diffraction theory by a finite difference method," USSR Computational Mathematics and Mathematical Physics, vol. 8, no. 5, pp. 282-288, 1968.

[3] R. H. Hardin and F. D. Tappert, "Applications of the split-step Fourier method to the numerical solution of nonlinear and variable coefficient wave equations," SIAM Review, vol. 15, no. 1, pp. 423-429, 1973.

[4] J. Kuttler and G. D. Dockery, "Theoretical description of the parabolic approximation/Fourier split-step method of representing electromagnetic propagation in the troposphere," Radio Science, vol. 26, no. 02, pp. 381393, 1991.

[5] M. Levy, Parabolic Equation Methods for Electromagnetic Wave Propagation. IET editions, 2000.

[6] H. Zhou, A. Chabory, and R. Douvenot, "Comparisons of discrete and continuous propagators for the modelling of low tropospheric propagation," in 11th European Conference on Antennas and Propagation (EuCAP), March 2017, pp. 1236-1238.

[7] — , "A 3-D split-step Fourier algorithm based on a discrete spectral representation of the propagation equation," IEEE Transactions on Antennas and Propagation, vol. 65, no. 4, pp. 1988-1995, April 2017.

[8] W. C. Chew, "Electromagnetic theory on a lattrice," Journal of Applied Physics, vol. 75, no. 10, pp. 4843-4850, 1994.

[9] F. L. Teixeira and W. C. Chew, "Lattice electromagnetic theory from a topological viewpoint," Journal of Mathematics and Physics, vol. 40, no. 1, pp. 169-187, 1999.

[10] D. Gabor, Theory of Communication. Institution of Electrical Engineering, 1946.

[11] A. Bogush and R. Elkins, "Gaussian field expansions for large aperture antennas," IEEE Transactions on Antennas and Propagation, vol. 34 no. 2, pp. 228-243, 1986.

[12] D. Lugara, C. Letrou, A. Shlivinski, E. Heyman, and A. Boag, "Framebased Gaussian beam summation method: Theory and applications," Radio Science, vol. 38, no. 2, 2003.

[13] J. J. Maciel and L. B. Felsen, "Systematic study of fields due to extended apertures by Gaussian beam discretization," IEEE transactions on antennas and propagation, vol. 37, no. 7, pp. 884-892, 1989.

[14] _ , "Discretized Gabor-based beam algorithm for time-harmonic radiation from two-dimensional truncated planar aperture distributions .I. formulation and solution," IEEE Transactions on Antennas and Propagation, vol. 50, no. 12, pp. 1751-1759, Dec 2002.

[15] A. Chabory, J. Sokoloff, and S. Bolioli, "Novel Gabor-based Gaussian beam expansion for curved aperture radiation in dimension two," Progress In Electromagnetics Research, vol. 58, pp. 171-185, 2006. 
[16] C. L'Hour, V. Fabbro, A. Chabory, and J. Sokoloff, "2-D propagation modeling in inhomogeneous refractive atmosphere based on Gaussian beams part I: Propagation modeling," IEEE Transactions on Antennas and Propagation, vol. 67, no. 8, pp. 5477-5486, 2019.

[17] — " "2-D propagation modeling in inhomogeneous refractive atmosphere based on Gaussian beams part II: Application to radio occultation," IEEE Transactions on Antennas and Propagation, vol. 67, no. 8, pp. 5487-5496, 2019.

[18] S. G. Mallat, A Wavelet Tour of Signal Processing. Academic press, 1999.

[19] T. K. Sarkar, M. Salazar-Palma, and M. C. Wicks, Wavelet Applications in Engineering Electromagnetics. Artech House, 2002.

[20] A. Iqbal and V. Jeoti, "A split step wavelet method for radiowave propagation modelling in tropospheric ducts," in $R F$ and Microwave Conference (RFM), 2011 IEEE International. IEEE, 2011, pp. 67-70.

[21] _ _ "Numerical modeling of radio wave propagation in horizontally inhomogeneous environment using split-step wavelet method," in Intelligent and Advanced Systems (ICIAS), 2012 4th International Conference on, vol. 1. IEEE, 2012, pp. 200-205.

[22] H. Zhou, R. Douvenot, and A. Chabory, "Modeling the long-range wave propagation by a split-step wavelet method," Journal of Computational Physics, vol. 402, p. 109042, 2020.

[23] S. G. Mallat, "Multiresolution approximations and wavelet orthonormal bases of $\mathrm{L}^{2}(\mathrm{R})$," Transactions of the American Mathematical Society, vol. 315 , no. 1, pp. 69-87, 1989 .

[24] C. Van Loan, Computational Frameworks for the Fast Fourier Transform. Siam, 1992, vol. 10.

[25] T. Bonnafont, R. Douvenot, and A. Chabory, "Split-step wavelet propagation modelling using local operators," in 2019 URSI International Symposium on Electromagnetic Theory (EMTS), 2019, pp. 1-4.

[26] - "A speed up of split-step wavelet for the computation of long range propagation," in 2020 14th European Conference on Antennas and Propagation (EuCAP), 2020, pp. 1-5.

[27] G. D. Dockery and J. R. Kuttler, "An improved impedance-boundary algorithm for Fourier split-step solutions of the parabolic wave equation," IEEE Transactions on Antennas and Propagation, vol. 44, no. 12, pp. 1592-1599, 1996.

[28] T. Bonnafont, R. Douvenot, and A. Chabory, "A local split-step wavelet method for the long range propagation simulation in 2D." Radio Science, vol. 56, no. 2, 2021.

[29] T. Bonnafont, "Modeling the atmopsheric long-range electromagnetic waves propagation in 3D using the wavelet transform," Ph.D. dissertation, Université Toulouse 3, 2020.

[30] G. A. Deschamps, "Gaussian beam as a bundle of complex rays," Electronics Letters, vol. 7, no. 23, pp. 684-685, November 1971.

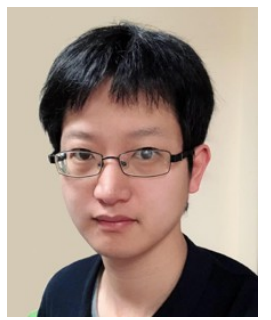

Hang ZHOU received the Engineer Degree in aerospace electronic from the École Nationale de l'Aviation Civile, Toulouse, France, in 2014 and the Ph.D. degree in aeronautical system and electromagnetics from Paul Sabatier University, Toulouse, in 2018. Since 2018, he has been a lecturer in the Sino-European Institute of Aviation Engineering of Civil Aviation University of China and a researcher of CAUC-ENAC joint Research Center of Applied Mathematics for Air Traffic Management, Civil Aviation University of China, Tianjin, China. His current research interests include electromagnetic theory and modeling, artificial intelligence theory and method, mainly for aeronautical applications.

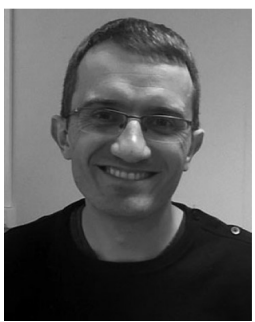

Alexandre CHABORY received the M.Sc. degree in electronic engineering from the French Civil Aviation University (ENAC), Toulouse, France, in 2001 $\mathrm{He}$ received the Ph.D. degree in electromagnetics from Paul Sabatier University, Toulouse, France, in 2004. From 2004 to 2007, he was a Postdoctoral Scientist with the Eindhoven University of Technology (TU/e), Eindhoven, The Netherlands. Since 2007, he has been an Assistant Professor with the Electromagnetics and Antennas Research Group, Telecom Laboratory, ENAC, Toulouse, France. Since 2012, he has been the head of this group. His research interests include electromagnetic theory and modeling, mainly for aeronautical applications.

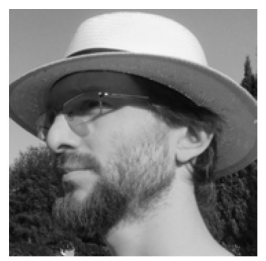

Rémi DOUVENOT was born in Chartres, France, in 1982. He received the engineer degree from the ENAC (French National Civil Aviation School), the M.Sc. from Toulouse University, France, in 2005, and the Ph.D. degrees in electrical engineering from Nantes University, France, in 2008. His Ph.D. was on refractivity from clutter. In 2009, he worked with the Laboratoire des Signaux et Syst'emes (L2S), UMR (SUPELEC - CNRS - Univ Paris-Sud) on non-destructive testing using eddy-currents. Since 2011, he has been a lecturer in electromagnetics with TELECOM/EMA research group, ENAC in Toulouse, France. His main research interests are the theory of wave propagation and its applications to radar and air transport systems. 